\title{
Design and Implementation of SFA Precious Old Film Resource Sharing Platform
}

\author{
Wanying Zhang ${ }^{1, a}$, Youdong Ding ${ }^{1,2}$, Bing $\mathrm{Yu}^{1}$, Biao Wu${ }^{1}$, Hang Zhao ${ }^{1,2}$ \\ ${ }^{1}$ Shanghai Film Academy, Shanghai University, Shanghai 200072 P.R. China \\ ${ }^{2}$ Shanghai Engineering Research Center of Motion Picture Special Effects, Shanghai University, Shanghai 200072 P.R. China
}

\begin{abstract}
When it comes to old films, we think the old films describe the story of the past normally. Many old films have been degraded or damaged for a long time. So the old films' restoration has been one of the mainstream film industry nowadays. It is because the old film is a record of the history and the old film resource is worth being cherished. At this point, it is particularly necessary and important to create a precious old film resource sharing platform. This platform runs on Eclipse environment and uses SSH framework structure, using MySQL as database, Apache tomcat as the server. Users can upload and download old films as well as the official administrator can manage the background functions. This platform can reserve valuable old film resource very well.
\end{abstract}

\section{Introduction}

An old film is a story about some specific moment like wars that describes the past years. It also records the growth of a country or nation. Namely, the old film highlights the traditional culture of a nation and shapes the soul of a society. The old film is carrying too many cultural heritage and historical truth and it is the country's precious cultural treasure. Figuratively speaking, losing an old film is like a tree leaving its root. So the restoration of old film has also become significantly. Some of the rebuilt films have been shown at the International Film Festival to remember the valuable experiences of predecessors. Some have been into local museums or landmark local buildings to show the public such as domestic and foreign tourists in order to carry forward the traditional culture. Others have been broadcast regularly in towns and villages to remind the old generations of the past days. But unfortunately, most old films have been kept in digital form all the time[1,2].

The old films are important for the older generation particularly. If we want to make the old films better infiltrate into the daily life of the public, it is crucial and indispensable to create a simple and convenient sharing platform of old film resources in order that people download them to watch daily. Of course, people also can upload the old films they have to the platform and share them with others. With a simple and convenient sharing platform, the public is willing to pay more attention to the development prospects and cultural effects of old films. Therefore, under this condition, making a precious old film resource sharing platform is the trend of the film media and also making a little contribution to the development of traditional culture.

\section{Platform design and analysis}

SFA ( Shanghai Film Academy) precious old film resource sharing platform is a convenient, popular and sharing information exchange platform. It is created in order to treasure the old film resources and share the old film resources. It can provide a convenient way and simple operation to increase the public attention of the old films and make a little contribution to carry forward the traditional culture.

The whole function includes two parts: the design and implementation of the front VIP user and the backstage management. The main function of the homepage is to register, upload and download the old film resources. The purpose is to have a good interaction among the users and to promote the role of the old movies. The backstage is mainly to manage the homepage functions and the whole system platform by the official administrators so that the platform can be carried out steadily for users.

\subsection{The whole design framework}

The whole design framework is shown below such as Figure 1. It includes the general structure of this platform. 


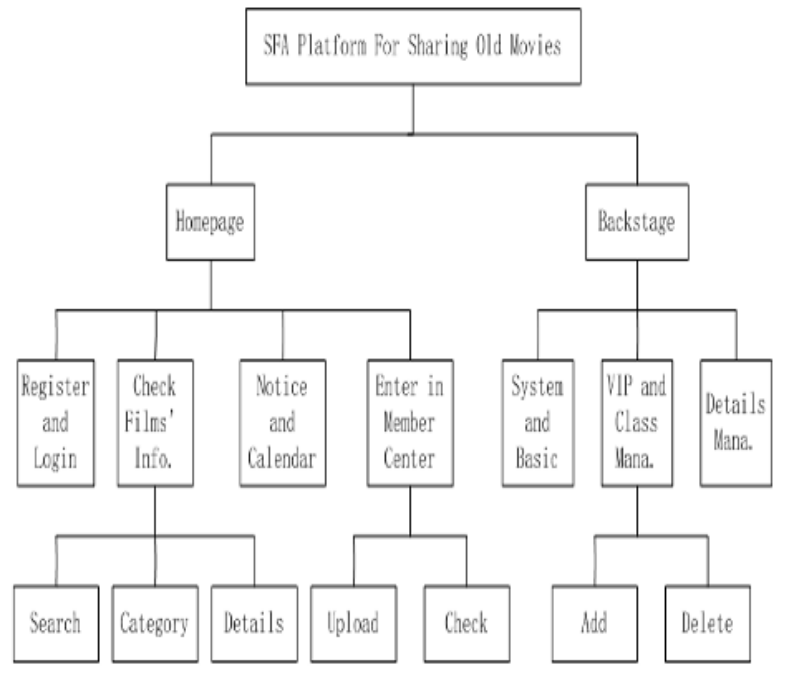

Figure 1. The whole framework of the platform.

\subsubsection{The homepage design}

The functions of the homepage design are shown as follows.

First, there are two basic operations about registering and logging. When visitors browse the platform, they can search for the old films and see the specific information. They cannot upload and download old film resources as well as publish messages except that they become VIP users through registration. During registration, visitors need to fill out personal information such as name, phone number and so on. After successful registration, all rights of members can be enjoyed.

Second, under the 'Film Information' module, it is also divided into three small modules, searching for the old film, looking at the catalogue classes of old films and the details of the old film. And there are some film classes of the old films on the homepage like $90 \mathrm{~s}, 80 \mathrm{~s}$, $70 \mathrm{~s}, 60,50 \mathrm{~s}, 40 \mathrm{~s}$ and before. All of VIP users can choose the old movies that they want to watch or upload an old movie according to the age.

Third, users can click 'VIP member center' to enter the user's member center. It can realize some functions like uploading old movies and checking the old movies' informations as well as collections.

In addition to the above functions, there are some small additional functions on the homepage. They are the latest official notices and real-time calendar.

Finally, users click on 'Log Out' and return to the homepage of platform.

\subsubsection{The backstage design}

The functions of the backstage design are shown as follows.

The official administrator enters the background homepage after entering the user name and password correctly. The front page shows the properties of the system directly, including the system store directory and so on.

First, the official administrator can change their password at any time in the 'Basic Operation' module, only need to enter their previous password once and new password two times. When the password coincides two times, the revision of the password will be successful. In addition, the official administrators also can add new administrators to facilitate better management of this platform.

Second, under the 'VIP Users Management' module, the official administrator can view all the members' information and manage them such as deleting users due to the improper operation of users and so on.

Third, the official administrator can add or delete the categories of old movies in the 'Film Class Management' module. The more detailed the classification, the more detailed it is, in order to better meet the needs of users. The administrator also can manage every old movie, such as checking the information of old movies and deleting anyone in the 'Film Management' module. But when the administrator is deleting an old movie, the administrator must fill out the reasons for the removal of this old movie, so as to make an open and fair share of the exchange and to ensure that all users are fair.

What's more, the official administrator can still add new notices or delete old notices, giving tips to users of the homepage for the latest news. Finally, the administrator clicks the 'Log Out' and return to the homepage.

\subsection{The database design}

During the process of database design, MySQL[3] is used as a database. MySQL is a relational database management system. It is one of the most popular relational database management systems. Five tables are designed, which are VIP users' information table, administrator information table, movie classification table, film information table and bulletin table. Each table has its name, alias, types, length and so on so that data can be stored more appropriately.

And using Navicat[4] as a visual database tool, it can modify data in real time to facilitate better management of database. Navicat is a fast, reliable and inexpensive database management tool designed to simplify database management and reduce system management costs. Navicat is built on intuitive graphical user interface, enabling you to create, organize, access and share information in a safe and simple way. There is just an example about films' catalogue like Figure 2.

\begin{tabular}{|c|l|l|l|l|l|}
\hline Alias & Types & Length & Decimal & Null & Primary key \\
\hline catalog_id & int & 11 & 0 & & P 1 \\
\hline catalog_name & varchar & 50 & 0 & J & \\
\hline catalog_description & text & 0 & 0 & J & \\
\hline catalog_delete & varchar & 50 & 0 & J & \\
\hline catalog_del-description & varchar & 500 & 0 & N & \\
\hline
\end{tabular}

Figure 2. Films' catalogue details.

\subsection{The Entity design}


The entity design[5-7] phase is based on the requirement analysis and designs various entities that they can meet the requirements, and the relationship between them, and lays the foundation for the later logical structure design. The entities designed here include user entity, administrator entity, old film classification and information entity, notice entity, etc. There is an example about user entity like Figure 3.

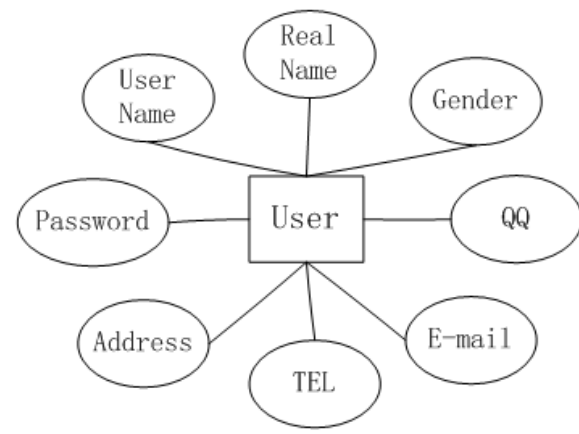

Figure 3. The user entity details.

\section{The Implementation of platform}

In the front of the platform, there is the main page of the entire platform and it is mainly based on simple and clear style. The main page, also called the homepage, is divided into following modules such as register and login module, search the movie module, check the notice and calendar module as well as view the movie information module[8-10]. The whole platform's homepage is shown as Figure 4.

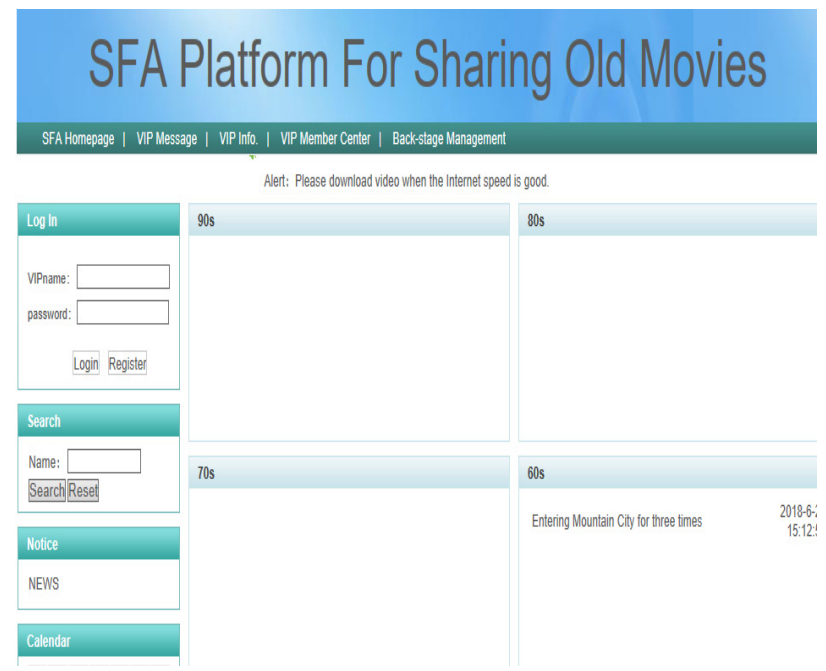

Figure 4. The SFA platform homepage.

Both users and visitors can click on the name of an old film to enter the detailed information page. It can show the name, cover poster, description, minutes and so on. VIP members can collect the old films and download old films to facilitate the view of communication. The old film's details is shown as Figure 5.

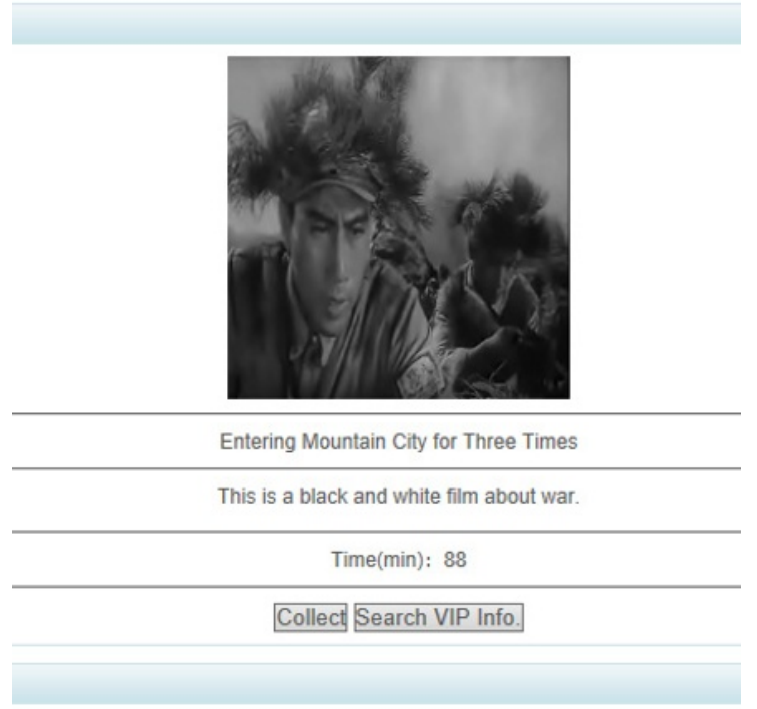

Figure 5. The old film's details.

Only platform's administrators can enter the backstage of the platform to manage it. Click the 'Back Stage Management' into the backstage. The backstage functions are shown as Figure 6. An example of film catalogue is shown such as Figure 7.

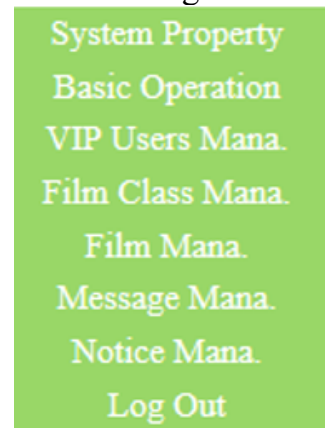

Figure 6. The backstage functions of platform.

\begin{tabular}{|c|c|c|c|}
\hline \multicolumn{4}{|l|}{ Class Catalog of Films } \\
\hline $\mathbb{D}$ & Class Name & Class Desceiption & Operation \\
\hline 109 & 908 & & Delete \\
\hline 110 & $80 \mathrm{~s}$ & & Delete \\
\hline 111 & $70 \mathrm{~s}$ & & Delete \\
\hline 112 & $60 \mathrm{~s}$ & & Delete \\
\hline 113 & $50 \mathrm{~s}$ & & Delete \\
\hline 114 & 40s and before & & Delete \\
\hline
\end{tabular}

Figure 7. The catalogue of platform.

\section{Results}

This platform has realized the function of the homepage interaction and the backstage management. Users can use it to share old film resource and communicate with each other. They can upload and download the old films they are interested. And the administrators also need to maintain the whole system in order that the platform runs safely and conveniently. The sharing platform uses the Java language for storing the old film resources on the SSH framework and runs on Eclipse environment. In addition, both the MySQL database and the Navicat visual tool have the powerful function to sustain the whole system's data files. This platform utilizes some computer technology to achieve the combination of the 
popular film art currently. With the continuous development of computer technology and multi-media technology, it is also applied to the field of art to complete the perfect combination of art and technology.

The old films are not only of profound significance for the elder generation, but also a valuable nourishment for the mind to enrich the cultural thoughts of the younger generation. It is hoped that the effective use of the platform can increase the public's awareness of the protection and promotion of the traditional culture. The status of the old film in the modern media is just like the status of the salt in food because they are ordinary and indispensable in daily life. This is the greatest respect and love for the old films.

\section{References}

1. H. Jiang, Audio-Visual 1, 38-39 (2018)

2. B. Wu, Y. Ding, R. Gong, TV technology 41, 110-116 (2017)

3. S. Tahaghoghi, H. E. Williams, Learning MySQL( Oreilly Media , 2018)

4. Tomorrow technology, Java Web from the beginning to mastery ( 2012)

5. S. Ma, G. Fang. Design and implementation of computer application system (Java) (2012)

6. S. Jia, J. Chen, G. Chen, Computer Application and Software3, 43-48 (2018)

7. Q. Liu, G. Liu, Wireless Interconnect Technology 02, 43-44 (2017)

8. F. Pan, S. Xu, Computer and Modernization2, 123-126 (2017)

9. W. Xia, Theoretical Research on Urban Construction 23, 57(2016)

10. J. Shen, G. Gu, Minitype Microcomputer System 23, 1449-1452 (2002) 